International Journal of Physical Research, $9(1)(2021) 42-48$
International Journal of Physical Research
SPC
Website: www.sciencepubco.com/index.php/IJPR
Research paper

\title{
On the compactification and reformation of string theory with three large atomic gravitational constants
}

\author{
U.V.S. Seshavatharam ${ }^{1 *}$, S. Lakshminarayana ${ }^{2}$ \\ ${ }^{1}$ Honorary faculty, I-SERVE, Survey no-42, Hitech city, Hyderabad-84, Telangana, INDIA \\ ${ }^{2}$ Dept. of Nuclear Physics, Andhra University, Visakhapatnam-03, AP, INDIA \\ *Corresponding author E-mail:seshavatharam.uvs@gmail.com
}

\begin{abstract}
Scientists strongly believe that, String theory is empowered with good mathematics and smartly fits gravity in unification program. Point to be noted is that, by considering the Planck length as characteristic amplitude associated with strings, String theory advances its ideological representation. Very unfortunate thing is that, even though, originally, String theory was proposed for understanding 'strong interaction', as Planck length is 20 orders of magnitude less than the nuclear size, it is badly failing in explaining and predicting nuclear scale physical phenomena. Here, we would like to stress the point that, within the scope of observed materialistic physical systems, without addressing the roots of $\mathrm{H}$-Bar and big G, it is impossible to construct a workable model of final unification. In this context, in our earlier publications, we proposed the existence of three large gravitational constants for the three atomic interactions. Based on the three large atomic gravitational constants, it is possible to have three different workable atomic Planck amplitudes and thus there is a possibility for reforming and compactifying the 10 dimensional String theory to $3+1$ dimensions. Proceeding further, H-bar can be shown to be a characteristic outcome of the electroweak interaction and big $\mathrm{G}$ can be shown to be a characteristic outcome of the three atomic gravitational constants.
\end{abstract}

Keywords: String Theory; Three Atomic Gravitational Constants; 3+1 Dimensions; 4G Model of Final Unification; Microscopic Quantum Gravity.

\begin{tabular}{|c|c|c|c|}
\hline \multicolumn{4}{|c|}{ List of symbols } \\
\hline 1) & Newtonian gravitational constant $=G_{N}$ & & \\
\hline 2) & Electromagnetic gravitational constant $=G_{e}$ & 16) & Mass of weak bosons $=\left(m_{z}\right)^{0},\left(m_{w}\right)^{ \pm}$ \\
\hline 3) & Nuclear gravitational constant $=G_{s}$ & 17) & Mass of electron $=m_{e}$ \\
\hline 4) & Weak gravitational constant $=G_{w}$ & 18) & Magnetic moment of proton $=\mu_{p}$ \\
\hline 5) & Fermi's weak coupling constant $=G_{F}$ & 19) & Weak interaction string tension $=F_{w}$ \\
\hline 6) & Strong coupling constant $=\alpha_{s}$ & 20) & Strong interaction string tension $=F_{s}$ \\
\hline 7) & Electroweak fermion $=M_{w}$ & 21) & Electromagnetic interaction string tension $=F$ e \\
\hline 8) & Reduced Planck's constant $=\hbar$ & 22) & Gravitational interaction string tension $=F_{g}$ \\
\hline 9) & Speed of light $=c$ & 23) & Weak interaction string potential $=E_{w}$ \\
\hline 10) & Elementary charge $=e$ & 24) & Strong interaction string potential $=E_{s}$ \\
\hline 11) & Strong nuclear charge $=e_{s}$ & 25) & Electromagnetic interaction string potential $=E_{e}$ \\
\hline 12) & Mass of proton $=m_{p}$ & 26) & Gravitational interaction string potential $=E_{g}$ \\
\hline 15) & Mass of neutron $=m_{n}$ & 27) & Fine structure ratio $=\alpha$ \\
\hline 14) & Mass of electron $=m_{e}$ & 28) & Nuclear fine structure ratio $=\alpha_{n}$ \\
\hline 15) & Mass of pions $=\left(m_{n}\right)^{0},\left(m_{n}\right)^{ \pm}$ & & \\
\hline
\end{tabular}

\section{Introduction}

Subject of final unification is very interesting and very challenging. Unifying gravity and quantum mechanics (QM) is very much complicated and scientists are trying their level best in different ways. As gravitational effects are negligible at quantum level, standard model of particle physics attempts to explore the secrets of elementary particles. On the other hand, as quantum effects are negligible at macroscopic level, General theory of relativity (GTR) attempts to explore the secrets of the universe. The most complicated question to be 
answered is - If celestial objects are confirmed to be made up of various kinds of atoms, whether 'gravity' is causing the atoms to form into celestial spheres or quantum rules are causing the atoms to form into celestial spheres that show gravity?

Astrophysics point of view or 'Planck scale' point of view, there is a possibility of observing the combined effects of GTR and QM at intermediate energy scales. In between GTR and QM, there exist fascinating and most complicated astrophysical objects, i.e. Black holes. Even though their detection is a great mystery, one can see the best possibility of understanding QM and GTR at extreme energy scales. Here, we would like to emphasize the point that, astrophysical observations pertaining to Black holes and various other compact stellar objects just reveal the combined effects of GTR and QM but no way indicating the secrets of unification of QM and GTR. One most common point of QM and GTR is "mass". By understanding the massive origin of elementary particles, it may be possible to probe the secrets of QM and GTR.

The primary goal of quantum gravity is to join the laws of quantum mechanics with the laws of general relativity into a single mathematically consistent framework. Many scientists believe that, String theory (Spenta Wadia 2008, Sunil Mukhi 2011) is one best candidate of quantum gravity. It is embedded with beautiful physical concepts like open strings, closed strings, string vibrations, string length, string tension and 'fermion-boson super symmetry'. Scientists strongly believe that, String theory is empowered with good mathematics and smartly fits gravity in unification program. Point to be noted is that, by considering the Planck length as characteristic amplitude associated with strings, String theory advances its ideological representation. Very unfortunate thing is that, even though, originally, String theory was proposed for understanding 'strong interaction', as Planck length is 20 orders of magnitude less than the nuclear size, it is badly failing in explaining and predicting nuclear scale physical phenomena. Here we would like to stress the point that, the main reason for its fatal failure is - "implementation of the two famous physical constants H-bar and big G as-they-are". We would like to say that, without addressing the roots of $\mathrm{H}-\mathrm{Bar}$ and big $\mathrm{G}$, it is impossible to construct a workable model of final unification.

\section{Extra dimensions and inadequacy of string theory}

The mathematics used in superstring theory requires at least 10 dimensions. String theorists believe that extra dimensions are wrapped up in the curled-up space first described by Kaluza and Klein (Kaluza 1921, Klein 1926, Randall and Sundrum1999, Stoica 2016). We would like to emphasize the following points.

1) The major inadequacy of String theory is - Extra dimensions. When there is no experimental evidence for extra dimensions, building a fundamental theory based on extra dimensions seems to be ad-hoc, misleading and speculative. Instead of extra dimensions, various utmost basic physical properties like mass, charge, density, radius, binding energy, magnetic moment, melting temperature, boiling temperature and solidification temperature can be considered as various angles of assessing the applicability of any fundamental theory.

2) Logically speaking, if $3+1$ dimensions demonstrate 4 different basic interactions, $9+1$ dimensions can have a minimum of 10 different new interactions. Clearly speaking, instead of finding unified solutions for $3+1$ dimensional basic interactions, unknowingly scientists are falling in a much more complex situation.

3) The basic question to be answered is, when the four basic interactions are being well operated in $3+1$ dimensions simultaneously, what is the necessity of introducing extra dimensions in understanding their unification scheme? When nobody is clear about the basics and existence of $5^{\text {th }}$ dimension, it is ad hoc to say that, electromagnetism and gravity seems to have similar behaviour in $5^{\text {th }}$ dimension.

4) Since 1920, no experiment has given a single (direct or indirect) clue for the existence of $5^{\text {th }}$ dimension (https://gizmodo.com/isthere-a-fifth-dimension-1832939412). By comparing the measurements of gravitational waves and light as they propagated through space, physicists at Laser interferometer gravitational-wave observatory LIGO (Barish Barry and Weiss Rainer 1999) were able to determine whether these two different waveforms were experiencing the same number of dimensions of spacetime. According to their experimental study (Kris Pardo et al.2018), the measurements of the neutron star collision suggest that both gravitational waves and particles of light experience four dimensions (three spatial dimensions plus time). Based on this inference, it seems possible to say that, evidence for the existence of higher dimensions is poor. If so, guessing about the existence of 10 to 26 dimensions may not be a right choice at fundamental level.

5) When nobody knows a single individual physical property of any $4+1$ dimensional physical system, discussing and studying about extra-dimensions is illogical.

6) So far, currently believed String theory models did not shed light on uncompactification of hidden multi-dimensions and acquiring of mass in $3+1$ dimensions.

7) When there is no theoretical procedure for indentifying $4+1$ dimensional physical phenomena, trying to identify any unknown $4+1$ dimensional physical phenomenon with an apparatus or equipment predesigned with $3+1$ dimensional procedure is another ad-hoc idea. If there is any possibility of inferring something from any such experiment, it needs a careful analysis with reference to an un-identified or new $3+1$ dimensional physical phenomenon.

8) When existence of any extra-dimensional physical system is uncertain, it is impossible to assess or confirm the reality of mathematics associated with its study.

9) There is no guarantee that, current laws of $3+1$ dimensional physics will work in extra-dimensions.

10) When $\mathrm{H}-\mathrm{Bar}$ and big $\mathrm{G}$ are confirmed to be practically associated with $3+1$ dimensions, applying them to unidentified extra dimensional physical models seem to be misguiding. Fundamental question to be answered is - How the magnitudes of $3+1$ physical constants are getting modified in extra-dimensions?- As nobody has witnessed any extra dimensional physical system, this question cannot be answered and cannot be ignored.

11) Strings and string properties can also be studied with $3+1$ dimensions.

12) When wave nature of particle is well established experimentally, particle waves can be considered as a characteristic representation of vibrating and moving strings.

13) No one can be against to the idea of multi universes but extra-dimensions may not be practical.

\section{Three large gravitational coupling constants}

When mass of any elementary particle is extremely small/negligible compared to macroscopic bodies, highly curved microscopic spacetime can be addressed with large gravitational constants and magnitude of elementary gravitational constant seems to increase with decreasing mass and increasing interaction range. Based on this logic, we consider the possibility of existence of three large gravitational 
constants assumed to be associated with the electromagnetic, strong and weak interactions (Tennakone1974, Sivaram \& Sinha 1977, De Sabbata \& Gasperini 1979, Salam, Sivaram 1993, Roberto Onofrio 2013, Seshavatharam \& Lakshminarayana 2010, 2011, 2015, 2017, 2019, 2020a, 2020b, 2020c, 2020d, 2020e). Compared to multi-dimensions and unproved maths of any String theory model, our proposal can be given some positive consideration. Following the notion of string theory, compactification of un-observable spatial dimensions might be playing a key role in hiding the large magnitudes of the three atomic gravitational constants. If multi dimensional physics is having a real sense, then, compactification of large magnitudes of atomic gravitational constants can also be possible.

By following our idea, in analogy with Planck scale, as an immediate result, it seems possible to have three different string amplitudes corresponding to electromagnetic, strong and weak interactions. In this way, String theory can be shaped to a model of elementary particle physics associated with $3+1$ dimensions. Another advantage is that, considering the combined effect of the three atomic gravitational constants, origins of $\mathrm{H}-\mathrm{Bar}$ and big $\mathrm{G}$ can be understood.Including the Newtonian gravitational constant, as the subject under consideration deals with 4 different gravitational constants, our model can be called as $4 \mathrm{G}$ model of final unification or Microscopic Quantum Gravity. With further study, Planck scale and electroweak scale can be studied in a unified manner. During cosmic evolution, if one is willing to give equal importance to Higgs boson and Planck mass in understanding the massive origin of elementary particles, then it seems quite logical to expect a common relation between Planck scale and Electroweak scale.

\section{Our basic assumptions}

We propose the following four assumptions.

1) Each atomic interaction is associated with a characteristic gravitational coupling constant and the corresponding values are,

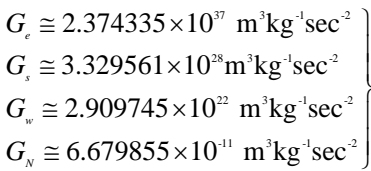

2) There exists a strong interaction elementary charge $\left(e_{s}\right) \cong 2.9463591 e$ in such a way that, it's squared ratio with normal elementary charge is close to reciprocal of the strong coupling constant

3) There exists a characteristic electroweak fermion of rest energy, $M_{w} c^{2} \cong 584.725 \mathrm{GeV}$. It can be considered as the zygote of all elementary particles.

4) Fermion-Boson mass ratio is 2.27 (Seshavatharam UVS \& Lakshminarayana 2010,2011,2020d).

\section{Inferences and consequences of above four assumptions}

Readers are encouraged to see our recently published papers (Seshavatharam \& Lakshminarayana 2015, 2020a, 2020b, 2020c, 2020d, 2020e) for possible introduction, and applications. Most important relations can be expressed as follows. We have made an attempt to derive them in a semi empirical approach (Seshavatharam \& Lakshminarayana 2020f).

$$
\frac{m_{p}}{m_{e}} \cong\left(\frac{G_{s} m_{p}^{2}}{\hbar c}\right)\left(\frac{G_{e} m_{e}^{2}}{\hbar c}\right) \cong\left(\frac{e_{s}^{2}}{4 \pi \varepsilon_{0} G_{s} m_{p}^{2}}\right) /\left(\frac{e^{2}}{4 \pi \varepsilon_{0} G_{e} m_{e}^{2}}\right)
$$

$$
G_{F} \cong G_{w} M_{w}^{2} R_{w}^{2}
$$$$
\text { where, } R_{w} \cong\left(2 G_{w} M_{w} / c^{2}\right)
$$

$$
m_{e} \cong\left(\frac{G_{w}}{G_{s}}\right) M_{w}
$$

$$
m_{p} \cong\left(\frac{G_{s}^{2}}{G_{w} G_{e}}\right) M_{w}
$$

$$
\frac{m_{p}}{m_{e}} \cong \frac{G_{s}^{3}}{G_{w}^{2} G_{e}}
$$

$$
\hbar c \cong G_{w} M_{w}^{2} \cong\left(\frac{G_{c} G_{w}}{G_{s}}\right) m_{p} m_{e} \cong G_{s} M_{w} m_{e}
$$

$$
\left(\frac{e_{s}}{e}\right) \cong \frac{G_{s} m_{p}^{2}}{G_{w} M_{w}^{2}} \cong \frac{G_{s}^{5}}{G_{e}^{2} G_{w}^{3}}
$$

$$
\left(\frac{e s}{e}\right)^{2} \cong \frac{1}{\alpha_{s}} \cong \frac{G_{s}^{10}}{G_{e}^{4} G_{w}^{6}}
$$




$$
G_{N} \cong \frac{G_{w}^{21} G_{e}^{10}}{G_{s}^{30}} \cong \frac{16 \pi^{4}}{\alpha^{2}}\left(\frac{m_{e}}{m_{p}}\right)^{14}\left(\frac{\hbar c}{m_{p}^{2}}\right)
$$

\section{Applications ofassumption-1}

'String tension' (Gibbons 2002) is a practical aspect of String theory. Considering the proposed three atomic gravitational constants and following the universal applicability of 'speed of light', approximate tensions associated with weak, strong, electromagnetic and gravitational interactions can be represented by,

$$
\left\{\begin{array}{l}
F_{w} \cong\left(\frac{c^{4}}{4 G_{w}}\right) \cong 6.94 \times 10^{10} \mathrm{~N} \\
F_{s} \cong\left(\frac{c^{4}}{4 G_{s}}\right) \cong 6.065 \times 10^{4} \mathrm{~N} \\
F_{c} \cong\left(\frac{c^{4}}{4 G_{c}}\right) \cong 8.505 \times 10^{-5} \mathrm{~N} \\
F_{s} \cong\left(\frac{c^{4}}{4 G_{N}}\right) \cong 3.026 \times 10^{43} \mathrm{~N}
\end{array}\right\}
$$

Following the universal applicability of 'elementary charge', approximate (operating) energy potentials associated with above string tensions can be represented by,

$$
\left\{\begin{array}{l}
E_{w} \cong \sqrt{\frac{e^{2}}{4 \pi \varepsilon_{0}}\left(\frac{c^{4}}{4 G_{w}}\right)} \cong 25.0 \mathrm{GeV} \\
E_{s} \cong \sqrt{\frac{e^{2}}{4 \pi \varepsilon_{0}}\left(\frac{c^{4}}{4 G_{s}}\right)} \cong 23.3 \mathrm{MeV} \\
E_{e} \cong \sqrt{\frac{e^{2}}{4 \pi \varepsilon_{0}}\left(\frac{c^{4}}{4 G_{e}}\right)} \cong 874 \mathrm{eV} \\
E_{g} \cong \sqrt{\frac{e^{2}}{4 \pi \varepsilon_{0}}\left(\frac{c^{4}}{4 G_{N}}\right)} \cong 8.355 \times 10^{7} \mathrm{~J}
\end{array}\right\}
$$

These estimated weak, strong and electromagnetic energy potentials seem to be close to experimental values.

\section{To validate assumption-3}

To validate assumption-3, we argue with the following nuclear and particle level observations.

1) It is generally believed that, $\left(m_{\pi}\right)^{0},\left(m_{\pi}\right)^{ \pm}$are the force carriers of strong interaction and $\left(m_{z}\right)^{0},\left(m_{w}\right)^{ \pm}$are the force carriers of weak interaction. Considering Pions and electroweak bosons, to a great surprise, we noticed that, $\left(\frac{\sqrt{m_{p} m_{n}}}{M_{w}}\right) \cong 0.001606 \cong\left(\frac{\sqrt{\left(m_{n} c^{2}\right)^{0}\left(m_{\pi} c^{2}\right)^{ \pm}}}{\sqrt{\left(m_{z} c^{2}\right)^{0}\left(m_{w} c^{2}\right)^{ \pm}}}\right) \cong\left(\frac{\sqrt{134.98 \times 139.57} \mathrm{MeV}}{\sqrt{80379.0 \times 91187.6} \mathrm{MeV}}\right) \cong 0.0016032$

2) It is also very interesting to note that, $\frac{\sqrt{m_{p} m_{n}}}{\sqrt{\left(m_{\pi}\right)^{0}\left(m_{\pi}\right)^{ \pm}}} \cong 6.84 \cong \frac{M_{w}}{\sqrt{\left(m_{z}\right)^{0}\left(m_{w}\right)^{ \pm}}} \cong 6.83$.

3) As neutron's weak decay is directly responsible for nuclear stability associated with beta emission, based on the two numerical coincidences, i.e. 0.0016 and 6.83 , existence of our assumed $584.725 \mathrm{GeV}$ weak fermion can be confirmed and it is also possible to have a relation of the form, $M_{w} \cong\left(\frac{\sqrt{\left(m_{z}\right)^{0}\left(m_{w}\right)^{ \pm}}}{\sqrt{\left(m_{\pi}\right)^{0}\left(m_{\pi}\right)^{ \pm}}}\right) m_{p} \cong 585.244 \mathrm{GeV} / c^{2}$.

4) With reference to nucleons and pions, it is reasonable to argue that, if one is willing to consider $\left(m_{z} c^{2}\right)^{0} \&\left(m_{w} c^{2}\right)^{ \pm}$as the force carriers of weak interaction (Fermi 1933,Englert \& Brout 1964, Higgs 1964), then, one should not ignore the possibility of considering the proposed weak fermion of rest energy $584.725 \mathrm{GeV}$ as the characteristic field generator of weak interaction.

5) Weak force carriers cannot exist without the existence of their weak field generating fermion.

\section{Understanding proton's root mean square radius}

Using the proposed strong nuclear charge $e_{s}$, proton magnetic moment $\mu_{p} \cong \frac{e_{s} \hbar}{2 m_{p}} \cong \frac{e G_{s} m_{p}}{2 c} \cong 1.488 \times 10^{-26} \mathrm{~J} /$ Tesla , nuclear fine structure ratio $\alpha_{n} \cong \frac{e_{s}^{2}}{4 \pi \varepsilon_{0} \hbar c} \cong 0.063345$, neutron life time (Pattie Jr et al. 2018), $t_{n} \cong\left(\frac{G_{e}^{2} m_{n}^{2}}{G_{w}\left(m_{n}-m_{p}\right) c^{3}}\right) \cong 875 \mathrm{sec}$, unified nuclear binding energy coefficient (Seshavatharam \& Lakshminarayana 2019, 2020), $B_{0} \cong \frac{1}{2} \sqrt{\alpha \times \alpha_{n}}\left(m_{p} c^{2}\right) \cong 10.09 \mathrm{MeV}$ and Fermi gas model of nuclear potential $E_{F} \cong \sqrt{\alpha \times \alpha_{n}}\left(m_{p} c^{2}+m_{n} c^{2}\right) \cong 40.37 \mathrm{MeV}$ can be fitted. With reference to the current experimental values of root mean square radius of pro- 
ton, $(0.833 \pm 0.01) \mathrm{fm}$ (Bezginov et al. 2019) and $\left(0.831 \pm 0.007_{s u t} \pm 0.012_{s y s}\right) \mathrm{fm}$ ( Xiong et al. 2019), we noticed one interesting relation. It can be expressed as,

$$
R_{p} \cong \sqrt{\left(\frac{4 \pi \varepsilon_{0} \hbar^{2}}{e_{s}^{2} m_{p}}\right)\left(\frac{\hbar}{m_{p} c}\right)} \cong \sqrt{\frac{4 \pi \varepsilon_{0} \hbar^{3}}{e_{s}^{2} m_{p}^{2} c}} \cong 0.835 \mathrm{fm}
$$

In this relation,

a) $\left(\frac{4 \pi \varepsilon_{0} \hbar^{2}}{e_{s}^{2} m_{p}}\right) \cong 3.32 \mathrm{fm}$ can be inferred as the Bohr's model of probable distance of finding proton in the nuclear well where the operating charge is $e_{s} \cong 2.946 e$.

b) $\left(\frac{\hbar}{m_{p} c}\right) \cong 0.21 \mathrm{fm}$ can be considered as the reduced Compton wavelength of proton.

Based on relation (12),

$$
\left[\left(\frac{e_{s}^{2}}{4 \pi \varepsilon_{0} c}\right)\left(m_{p} R_{p} c\right)^{2}\right]^{\frac{1}{3}} \cong \hbar
$$

\section{Understanding nuclear binding energy with single energy coefficient and four simple terms}

We would like to emphasize the fact that, physics and mathematics associated with fixing of the energy coefficients of semi empirical mass formula (SEMF) (Royer \& Subercaze 2013, Möller et al. 2016, Cht Mavrodiev \& Deliyergiyev 2018, Xia et al. 2018) are neither connected with residual strong nuclear force nor connected with strong coupling constant $\alpha_{s}$. Since nuclear force is mediated via quarks and gluons, it is necessary and compulsory to study the nuclear binding energy scheme in terms of nuclear coupling constants. In this direction, N. Ghahramany and team members have taken a great initiative in exploring the secrets of nuclear binding energy and magic numbers (Ghahramany et al. 2011, 2012) with reference to quarks. Very interesting point of their study is that - nuclear binding energy can be understood with two or three terms having single variable energy coefficient. In this direction, based on three unified assumptions connected with gravity and atomic interactions, in a semi empirical approach, we have developed a very simple formula for nuclear binding energy with single energy coefficient having four simple terms (Seshavatharam \& Lakshminarayana 2020f) . Corresponding relations can be expressed in the following way. Starting from $\mathrm{Z}=3$ to 118 ,

$$
\begin{aligned}
A_{s} & \cong 2 Z+0.0064 Z^{2} \\
& \cong \text { Estimated mass number close to proton-neutron mean stability line. } \\
B_{A} & \cong\left\{A-(1+0.0019 A \sqrt{Z N})-A^{1 / 3}-\frac{\left(A_{s}-A\right)^{2}}{A_{s}}\right\}\left(B_{0} \cong 10.1 \mathrm{MeV}\right) \\
& \cong \text { Estimated nuclear binding energy }
\end{aligned}
$$

Here, we would like to appeal that,

1) The two numbers, 0.0064 and 0.0019 can be considered to be associated with fine structure, ratio $\alpha$ and strong coupling constant $\alpha_{s}$ with a relation of the form, $\left[\frac{\left(1-\alpha_{s}\right)^{n}}{2 n-1}\right] \alpha \cong(0.006457,0.0019)$ where, $n=1$ and 2.

2) $(1+0.0019 A \sqrt{Z N})$ can be called as the geometric number of free or unbound nucleons.

3) $A^{1 / 3}$ can be called as radial factor associated with nucleons.

4) $\frac{\left(A_{s}-A\right)^{2}}{A_{s}}$ can be called as isotopic asymmetric term associated with mean stable mass number.

5) Binding energy coefficient can be expressed in the following way.

$$
\begin{aligned}
& B_{0} \cong \frac{1}{\alpha_{s}}\left(\frac{e^{2}}{4 \pi \varepsilon_{0} R_{0}}\right) \cong\left(\frac{e_{s}^{2}}{4 \pi \varepsilon_{0} R_{0}}\right) \\
& \cong \frac{1}{2} \sqrt{\left(\frac{e^{2}}{4 \pi \varepsilon_{0} \hbar c}\right)\left(\frac{e_{s}^{2}}{4 \pi \varepsilon_{0} \hbar c}\right)}\left(m_{p} c^{2}\right) \cong 10.1 \mathrm{MeV}
\end{aligned}
$$

where

$$
\left\{\begin{array}{l}
\frac{1}{\alpha_{s}} \cong\left(\frac{e_{s}}{e}\right)^{2} \cong\left(\frac{G_{s} m_{p}^{2}}{\hbar c}\right)^{2} \cong \frac{1}{0.1151937} \\
\text { and } R_{0} \cong \frac{2 G_{s} m_{p}}{c^{2}} \cong 1.24 \text { fermi }
\end{array}\right\}
$$




\section{Understanding super symmetry in strong interaction}

Based on the beautiful concepts of Super symmetry, we tried our level best to explore Strong interaction in the view of the existence of Quark fermions and Quark bosons. We emphasize that, 1) Strong interaction is one best platform for observing and confirming super symmetry (SUSY); 2) All observed baryons and mesons are SUSY particles only; 3) SUSY particles exist at all energy scales and are within the reach of current accelerators. For details, readers are encouraged to see our recently published paper (Seshavatharam \& Lakshminarayana 2020d).

\section{Conclusion}

With further study, research and mathematical analysis, retaining the originality of physics, string theory models can be compactified to $3+1$ dimensions. In a progressive manner, by thoroughly exploring and collecting the possible experimental evidences for extra dimensions, future research can be carried out with ease.

\section{Acknowledgements}

Author Seshavatharam is indebted to professors shri M. Nagaphani Sarma, Chairman, shri K.V. Krishna Murthy, founder Chairman, Institute of Scientific Research in Vedas (I-SERVE), Hyderabad, India and Shri K.V.R.S. Murthy, former scientist IICT (CSIR), Govt. of India, Director, Research and Development, I-SERVE, for their valuable guidance and great support in developing this subject.

\section{References}

[1] Barish Barry C and Weiss Rainer. (1999). LIGO and the Detection of Gravitational Waves. Physics Today. 52 (10), 44. https://doi.org/10.1063/1.882861.

[2] Bezginov N. et al. (2019). A measurement of the atomic hydrogen Lamb shift and the proton charge radius. Science, 365(6457), 1007. https://doi.org/10.1126/science.aau7807.

[3] ChtMavrodiev S and Deliyergiyev MA.(2018). Modification of the nuclear landscape in the inverse problem framework using the generalized Bethe-Weizsäcker mass formula. Int. J. Mod. Phys. E 27: 1850015. https://doi.org/10.1142/S0218301318500155.

[4] De Sabbata V and Gasperini M. Strong gravity and weak interactions. (1979). Gen. Relat. Gravit. 10( 9), 731-741. https://doi.org/10.1007/BF00756600.

[5] Englert F and Brout R. (1964). Broken Symmetry and the Mass of Gauge Vector Mesons. Physical Review Letters, 13(9), 321-323. https://doi.org/10.1103/PhysRevLett.13.321.

[6] Fermi E. (1933). Tentativo di unateoriadeiraggi $\beta$. La Ricerca Scientifica (in Italian). 2 (12).

[7] Ghahramany N, ShGharaati, Ghanaatian M, Hora H.(2011). New scheme of nuclide and nuclear binding energy from quark-like model. Iranian Journal of Science \& Technology. A3, 201-208.

[8] Ghahramany, N., Gharaati, S., \&Ghanaatian, M. (2012). New approach to nuclear binding energy in integrated nuclear model. Journal of Theoretical and Applied Physics, 6(1), 3. https://doi.org/10.1186/2251-7235-6-3.

[9] Gibbons G.W. (2002). The Maximum Tension Principle in General Relativity. Foundations of Physics. $32, \quad 1891$. https://doi.org/10.1023/A:1022370717626.

[10] Higgs P. (1964). Broken Symmetries and the Masses of Gauge Bosons. Physical Review Letters. 13 (16), 508-509. https://doi.org/10.1103/PhysRevLett.13.508.

[11] Kaluza T. (1921). On the problem of unity in physics. Sitzungsber. Preuss. Akad. Wiss. Berlin (Math. Phys.), 966-972.

[12] Klein O.(1926). Quantum theory and five-dimensional theory of relativity. Z. Phys., 37:895-906. https://doi.org/10.1007/BF01397481.

[13] Kris Pardo et al. (2018). Limits on the number of spacetime dimensions from GW170817. Journal of Cosmology and Astroparticle Physics. 7, 048. https://doi.org/10.1088/1475-7516/2018/07/048.

[14] Möller, P., Sierk, A. J., Ichikawa, T., Sagawa, H. (2016). Nuclear ground-state masses and deformations: FRDM 2012. Atomic Data and Nuclear Data Tables, 109, 1-204. https://doi.org/10.1016/j.adt.2015.10.002.

[15] Pattie Jr R.W. et al. (2018). Measurement of the neutron lifetime using a magneto-gravitational trap and in situ detection. Science. 360(6389): 627632.

[16] RandallL and Sundrum R. (1999). Large mass hierarchy from a small extra dimension. Phys. Rev. Lett., 83(17), 3370. https://doi.org/10.1103/PhysRevLett.83.3370.

[17] Roberto Onofrio.(2013). On weak interactions as short-distance manifestations of gravity. Modern Physics Letters A 28 , 1350022. https://doi.org/10.1142/S0217732313500223.

[18] Royer G and Subercaze A. (2013). Coefficients of different macro-microscopic mass formulae from the AME2012 atomic mass evaluation. Nuclear Physics A 917, 1-14. https://doi.org/10.1016/j.nuclphysa.2013.09.003.

[19] Salam A and Sivaram C. (1993). Strong Gravity Approach to QCD and Confinement. Mod. Phys. Lett., A8(4), 321-326. https://doi.org/10.1142/S0217732393000325.

[20] Seshavatharam UVS and Lakshminarayana S. (2010). Super Symmetry in Strong and Weak interactions. Int. J. Mod. Phys. E, 19(2), p.263-280. https://doi.org/10.1142/S021830131001473X.

[21] Seshavatharam UVS and Lakshminarayana S. (2011). SUSY and strong nuclear gravity in (120-160) GeV mass range. Hadronic journal, 34(3), 277.

[22] Seshavatharam UVS and Lakshminarayana S. (2015). Understanding nuclear binding energy with low energy nuclear elementary charge and root mean square radius of proton. Journal of Applied Physical Science International, 6(1),1-13.

[23] Seshavatharam UVS and Lakshminarayana S. (2017). Understanding the basics of final unification with three gravitational constants associated with nuclear, electromagnetic and gravitational interactions. Journal of Nuclear Physics, Material Sciences, Radiation and Applications 4(1),1-19. https://doi.org/10.15415/jnp.2017.42031.

[24] Seshavatharam UVS and Lakshminarayana S. (2019). On the role of four gravitational constants in nuclear structure. Mapana Journal of Sciences, 18(1), 21-45. https://doi.org/10.12723/mjs.48.2.

[25] Seshavatharam UVS and Lakshminarayana S. (2020a). Is reduced Planck's constant - an outcome of electroweak gravity? Mapana Journal of Sciences. 19(1), 1-13. https://doi.org/10.20944/preprints201912.0231.v2.

[26] Seshavatharam UVS, Lakshminarayana S. (2020b). Understanding nuclear stability and binding energy with powers of the strong coupling constant. Mapana Journal of Sciences. 19(1), 35-70.

[27] Seshavatharam UVS and Lakshminarayana S. (2020c). Implications and Applications of Fermi Scale Quantum Gravity. International Astronomy and Astrophysics Research Journal.2(1),13-30. 
[28] Seshavatharam UVS and Lakshminarayana S. (2020d). 4G Model of Fractional Charge Strong-Weak Super Symmetry. International Astronomy and Astrophysics Research Journal.2(1),31-55. https://doi.org/10.20944/preprints201912.0391.v1.

[29] Seshavatharam UVS and Lakshminarayana S. (2020e). Significance and Applications of the Strong Coupling Constant in the Light of Large Nuclear Gravity and Up and Down Quark Clusters. International Astronomy and Astrophysics Research Journal. 2(1):56-68. https://doi.org/10.20944/preprints201911.0398.v2.

[30] Seshavatharam UVS and Lakshminarayana S. (2020f). Semi Empirical Derivations Pertaining to 4G Model of Final Unification. International Astronomy and Astrophysics Research Journal 2(1), 69-74. https://doi.org/10.20944/preprints202001.0020.v1.

[31] Sivaram C and SinhaK (1977). Strong gravity, black holes, and hadrons. Physical Review D., 16(6), 1975-1978. https://doi.org/10.1103/PhysRevD.16.1975.

[32] Spenta R. Wadia. (2008). String theory: a framework for quantum gravity and various applications. Current Science. 95(9), 10.

[33] Stoica O.C. (2016). Kaluza theory with zero-length extra dimensions. Int. J. Mod. Phys. D, $25(11)$, 1640004. https://doi.org/10.1142/S0218271816400046.

[34] Sunil Mukhi. (2011). String theory: a perspective over the last 25 years. Class. Quant. Grav. 28 153001. https://doi.org/10.1088/02649381/28/15/153001.

[35] Tennakone T. (1974).Electron, muon, proton, and strong gravity. Phys. Rev. D, 10, 1722. https://doi.org/10.1103/PhysRevD.10.1722.

[36] Xia, X. W., Lim, Y., Zhao, P. W., Liang, H. Z., Qu, X. Y., Chen, Y., \& Meng, J. (2018). The limits of the nuclear landscape explored by the relativistic continuum Hartree-Bogoliubov theory. Atomic Data and Nuclear Data Tables, 121, 1-215. https://doi.org/10.1016/j.adt.2017.09.001.

[37] Xiong W et al. (2019). A small proton charge radius from an electron-proton scattering experiment. Nature .575, 147-150. https://doi.org/10.1038/s41586-019-1721-2. 